\title{
Nanomedicine Making Headway across the Blood Brain Barrier
}

\section{Agnes E. Ostafin ${ }^{1 *}$ and Michael M. Batenjany²}

${ }^{1}$ Nanomaterials Center, Nanolnstitute Chemical Engineering, Bioengineering, University of Utah, Sorenson Molecular Biotechnology Building \#5534, 36 South Wasatch Drive, Salt Lake City, UT 84112-5001, USA

${ }^{2}$ Nanoshell Company LLC, Research \& Development, 1747 E Beechwood Dr, Layton, UT 84040, USA

Nanotechnological advances implemented by nanomedicine have allowed significant development of imaging strategies, therapeutics and theranostics for many severe and life threatening diseases such as brain tumors, Alzheimer's disease, Parkinson's disease and other neurological disorders. The Blood-Brain Barrier (BBB) and the Blood-Cerebrospinal Fluid Barrier (BCSF) have hindered the effective delivery of brain imaging agents and therapeutics. However, within the last decade, innovations in the design of nanoparticles are proving to be very exciting.

Briefly, the blood brain barrier [1-3] is comprised of brain microvascular endothelial cells that are connected by tight junctions. The tight junctions are composed of transmembrane proteins, often dimers, and further anchored by a protein complex that includes zo-1 and associated proteins. In the human brain, there are approximately 100 billion microvascular capillaries, totaling 400 miles in length (roughly the length of Lake Superior). The tight junctions and some selective metabolic barriers/transporters are responsible for preventing, and/or restricting the passage of most compounds in blood into brain. Very small class of drugs or molecules with high lipid solubility and low molecular weight $(<500$ Daltons) can only cross the BBB. A limited set of nutrients (certain amino acids or glucose) and a restricted number of macromolecules (transferrin, lactoferrin, insulin) are allowed to enter through a complex set of transporters [3]. Brain endothelial cells also express a multidrug resistance protein (e.g. P-glycoprotein, etc.), which actively transports a variety of small molecules out of the cells [1]. In addition, and distinct from the BBB, is the blood cerebrospinal fluid barrier. The BCSF formed by cells of the choroid plexus controls transport of molecules between the blood and the cerebrospinal fluid (CSF). The BCSF contains mechanisms for facilitated diffusion and active transport, both into and out of the CSF [1]. Hence, the BBB and BCSF are formidable obstacles for delivery of imaging agents and therapeutics into the brain.

Non-invasive imaging techniques have included a vast number of methods including X-ray CT, MRI, PET, SPECT, as well as optical and optoacoustic methods. To this end, improved contrast for MRI scans can be achieved by using engineered nanoparticle (NP) and magnetic NP's (MNPs) [1]. In addition, supramagnetic iron oxide NPs, paramagnetic gadolinium and perfluorocarbons have also been utilized [1]. For example, Fiandaca et al. [4] used gadolinium-loaded liposomes and adeno-associated viral vectors to achieve real time MRI imaging to three regions of a non-human primate brain. Schemes using liposomeencapsulated hemoglobin have improved PET scans [5], while gold NPs have increased optoacoustic signals in vivo [6]. Caveats and design restrictions are still formidable as nanomedicine improves imaging and drug delivery for brain tumors [7].

Nanomedicine's impact on the therapeutic and theranostic treatment and assessment of brain tumors is probably most evident by considering transcytosis, liposomal formulations and various schemes utilizing dendrimers. Transcytosis can be Receptor Mediated (RMT) or Adsorptive Mediated (AMT), whereby nanoparticles are surface tagged with ligands that recognize specific receptors (e.g. Transferrin receptor), or by the presence of an excess positive charge, respectively
[7]. In general, large molecular size drug delivery favors RMT and has included liposomal forms. PEGylated liposomes conjugated to a murine monoclonal antibody (OX26 monoclonal antibody) to the rat transferrin receptor demonstrated a high degree of brain delivery [8]. Lu et al. [9] used adsorptive mediated transport to successfully deliver a plasmid incorporated into cationic albumin-conjugated PEGylated NP's, as gene therapy in gliomas of balb/c mice. Many schemes use dendrimers. For example, Janaszewska et al. [10] described the ability of maltotriose-modified poly(propylene imine) (PPI) dendrimers to cross the blood brain barrier. In fact, a whole issue of the New Journal of Chemistry has been devoted entirely to dendrimers [11]. Each of the above methods has advantages and disadvantages. These are related to cost, reproducibility, stability and safety $[1,7]$.

New innovations in nanomedicine are promising better imaging and drug delivery avenues to assess and treat many brain and CNS related pathologies. Indeed, the last decade has seen a significant number of innovations, which seem to promise the ability to better cross the BBB and offer hope for many severe and life threatening diseases of the brain and other neurological disorders. These formulations are, however, still at an early stage. Critical are safety studies, which thus far have been few. Ideally, one would achieve a safe, theranostic preparation, wherein one can directly monitor the efficacy concomitant with the treatment.

\section{References}

1. Bhaskar S, Tian F, Stoeger T, Kreyling W, de la Fuente JM, et al. (2010) Multifunctional Nanocarriers for diagnostics, drug delivery and targeted treatment across blood-brain barrier: perspectives on tracking and neuroimaging. Part Fibre Toxicol 7: 3.

2. Gandhi M, Bohra H, Daniel V, Gupta A (2010) Nanotechnology in Blood Brain Barrier: A Review. International Journal of Pharmaceutical \& Biological Archives 1: 37-43.

3. Jong A, Huang SH (2005) Blood-brain barrier drug discovery for central nervous system infections. Curr Drug Targets Infect Disord 5: 65-72.

4. Fiandaca MS, Varenika V, Eberling J, McKnight T, Bringas J, et al. (2009) Realtime MR imaging of adeno-associated viral vector delivery to the primate brain Neuroimage 47: T27-T35.

5. Urakami T, Kawaguchi AT, Akai S, Hatanaka K, Koide H, et al. (2009) In vivo distribution of liposome-encapsulated hemoglobin determined by positron emission tomography. Artif Organs 33: 164-168.

*Corresponding author: Agnes E. Ostafin, Nanomaterials Center, Nanolnstitute Chemical Engineering, Bioengineering, University of Utah, Sorenson Molecular Biotechnology Building \#5534, 36 South Wasatch Drive, Salt Lake City, UT 84112 5001, USA, Tel: 801-581-3413; Fax : 801-581-4816; E-mail: a.ostafin@utah.edu

Received October 19, 2012; Accepted October 20, 2012; Published October 23 2012

Citation: Ostafin AE, Batenjany MM (2012) Nanomedicine Making Headway across the Blood Brain Barrier. J Nanomed Nanotechol 3:e123. doi:10.4172/21577439.1000e123

Copyright: (c) 2012 Ostafin AE, et al. This is an open-access article distributed under the terms of the Creative Commons Attribution License, which permits unrestricted use, distribution, and reproduction in any medium, provided the original author and source are credited. 
Citation: Ostafin AE, Batenjany MM (2012) Nanomedicine Making Headway across the Blood Brain Barrier. J Nanomed Nanotechol 3:e123. doi:10.4172/2157-7439.1000e123

Page 2 of 2

6. Ungureanu C, Van Weperen T, Sijl J, Rayavarapu R, Manohar S, et al. (2008) Acoustic signals from gold nanoparticles irradiated with pulsed lasers. Journal of the Acoustical Society of America 123: 3370.

7. Chapman M, Pascu S (2012) Nanomedicines Design: Approaches towards the Imaging and Therapy of Brain Tumours. J Nanomedic Nanotechnol S4.

8. Schnyder A, Huwyler J (2005) Drug transport to brain with targeted liposomes. NeuroRx 2: 99-107.
9. Lu W, Sun $Q$, Wan J, She Z, Jiang XG (2006) Cationic albumin-conjugated pegylated nanoparticles allow gene delivery into brain tumors via intravenous administration. Cancer Res 66: 11878-11887.

10. Janaszewska A, Ziemba B, Ciepluch K, Appelhans D, Voit B, et al. (2012) The biodistribution of maltotriose modified poly(propylene imine) (PPI) dendrimers conjugated with fluorescein-proofs of crossing blood-brain-barrier. New J Chem 36: 350-353.

11. (2007) Regulating nanomedicine. Nat Mater 6: 249. 